\title{
The Effect of Extracorporeal Shock Wave Therapy in the Treatment of Patients with Trigger Finger
}

This article was published in the following Dove Press journal:

Open Access Journal of Sports Medicine

\section{Babak Vahdatpour (D) \\ Fahimeh Momeni \\ Ali Tahmasebi \\ Parisa Taheri (D)}

Department of Physical Medicine and Rehabilitation, Isfahan University of Medical Sciences, Isfahan, Iran
Correspondence: Parisa Taheri Isfahan University of Medical Sciences, School of Medicine, Department of Physical Medicine and Rehabilitation, Hezar-Jarib Avenue, Isfahan, Iran Email Prs_taheri@yahoo.com
Introduction: Trigger finger disorder is a sudden release or locking of a finger during flexion or extension. Regarding the complications and disadvantages mentioned for the methods used in the treatment of trigger finger disorder, the aim of this study was to investigate the effect of extracorporeal shock wave therapy in the treatment of patients with trigger finger.

Methods: This study was an interventional study recruiting 19 patients with trigger finger disorder. Evaluation of pain severity, severity of triggering, and functional impact of triggering was carried out using the Visual Analogue Scale, Trigger Finger Score suggested by Quinnell, and Quick-Disabilities of the Arm, Shoulder, and Hand (DASH) questionnaire, respectively, before intervention, immediately after intervention, and in 6 and 18 weeks after intervention. Each patient was treated with extracorporeal shock wave therapy in three sessions with a 1-week interval. Data were analyzed in Statistical Package for the Social Sciences (SPSS) software using ANOVA to monitor changes in pain severity, severity of triggering, and functional impact of triggering during follow-ups.

Results: There were statistically significant differences with regard to reduction of the pain severity, severity of triggering, and functional impact of triggering before intervention, immediately after intervention, and in 6 and 18 weeks after intervention $(P<0.01)$. However, the effect of extracorporeal shock wave therapy on reducing severity of triggering immediately after intervention did not yield a statistically significant difference compared to before intervention $(P>0.01)$.

Conclusion: It seems that extracorporeal shock wave therapy leads to a reduction in pain severity, severity of triggering, and functional impact of triggering. These effects persisted until the 18th week after the intervention. It is recommended to use extracorporeal shock wave therapy in terms of a non-invasive intervention with no significant complications for patients with trigger finger.

Keywords: extracorporeal shock wave therapy, trigger finger, pain severity, severity of triggering, functional impact of triggering

\section{Introduction}

Trigger finger disorder, also known as stenosing tenovaginitis, is a sudden release or locking of a finger during flexion or extension, ${ }^{1,2}$ which is due to hypertrophy at the intersection of the tendon with the tendon pulley and prevents normal forward and backward gliding of the tendon under the pulley. It is postulated that this disorder is caused by high pressure at the proximal edge of the A1 pulley and the discrepancy between the diameter of the flexor tendon and its sheath at the metacarpal head. ${ }^{2}$ The link between this disorder and repeated trauma is frequently highlighted in scholarly articles; ${ }^{2}$ however, the main etiology is still unclear. ${ }^{1}$ 
Thumb (33\% in adults and 90\% in children) followed by ring finger $(27 \%)$ are the most commonly involved fingers. ${ }^{2,3}$ Moreover, the right hand is more frequently involved compared to the left hand ${ }^{4,5}$ and the dominant hand is more frequently involved compared to the nondominant hand. ${ }^{3}$ The age distribution of trigger finger is showing a pattern that includes children under the age of 6 years and adults over 40 years old, especially women aged between 50 and 60 years old. ${ }^{4}$ The prevalence of trigger finger is higher in patients with rheumatoid arthritis ${ }^{2}$ and diabetes (with a prevalence of $10 \%$ to $20 \%$ ) ${ }^{6,7}$ compared to the normal population (with a prevalence of $2 \%$ to $3 \%)^{7}$ Additionally, patients with rheumatoid arthritis and diabetes can experience trigger finger in multiple fingers., ${ }^{2,7}$ Other conditions associated with the disease include carpal tunnel syndrome, osteoarthritis, de quervain's tenosynovitis, and hypothyroidism. ${ }^{8-11}$

Diagnosis is completely based on history and clinical examination, and no paraclinical examination is required without a history of trauma or inflammatory arthritis. ${ }^{2,12}$ In history taking, the patient is typically complainant about pain in the PIP joint, morning stiffness, and tenderness on the A1 pulley; however, the main pathology is in the MCP joint. In more severe cases, the patient may have a history of a locking finger that could be resolved either passively or actively. ${ }^{1,2}$ The pain and locking of the finger caused by the disease will result in functional limitation. ${ }^{2}$

Treatments for this disease are diverse and include NSAIDs, cryotherapy, hand splints, corticosteroid injections, percutaneous or open surgery, ${ }^{1}$ and musculoskeletal ultrasound. ${ }^{13,14}$ Selected treatment for each patient depends on the severity of triggering (the more severe cases respond better to injection), the onset of triggering, previous treatment, the patient's level of activity, and the patient's and therapist's preferences. ${ }^{1,2}$ The proposed splint holds the MCP joint at $0^{\circ}$ of extension ${ }^{1}$ or $10^{\circ}$ to $15^{\circ}$ of flexion for up to 3 to 6 weeks. ${ }^{2}$ The splint was not effective in patients with multiple finger involvement. The relapse rate with this treatment was $12 \%$ over a year. ${ }^{15}$ Corticosteroid injection was less effective in patients with multiple finger involvement, such as diabetic and rheumatoid patients, and in those with symptoms lasting longer than 4 months. $^{2,16}$ Tendon rupture is more likely in patients with rheumatoid arthritis after corticosteroid injection and repeated injections are not recommended. ${ }^{2}$ For other patients, the maximum number of injections is up to three injections according to the new European guidelines. ${ }^{1}$ Surgery is indicated in the case of injection failure. Patients with diabetes, rheumatoid arthritis, multiple finger involvement, and lower ages will require surgery at the start of treatment. Complications of this type of treatment are infection, nerve injury, and bowing of flexor tendons. ${ }^{2}$ Musculoskeletal ultrasound has also gained an important place in the diagnosis of and intervention for musculoskeletal disorders, including trigger finger, due to being inexpensive, non-invasive, repeatable, convenient, not requiring any exposure to radiation, and providing dynamic imaging. ${ }^{13,14}$

The extracorporeal shock waves are defined as sound waves that can maximize the pressure of the target tissue over a few nanoseconds. ${ }^{17}$ Recently, extracorporeal shock wave therapy (ESWT) has been proposed as an alternative treatment in patients not responding to conservative treatments. This method has been used in orthopedic diseases such as plantar fasciitis, lateral epicondylitis, calcific shoulder tendonitis, non-union of long bones, ${ }^{17-22}$ patellar tendinopathy, femoral head avascular necrosis, and carpal tunnel syndrome. The success rate of this method varies between $65 \%$ and $91 \%$ in the treatment of these diseases in various studies, while the complications are low and can be neglected.

The shock wave has two focus and radial types, which do not have a focal point in the radial type and can be used without specifying painful points. The use of this method has been approved by the FDA for plantar fasciitis and lateral epicondylitis. ${ }^{17}$ The contraindications of this method include hemorrhagic diseases and pregnancies. Its complications in low to moderate energies include femoral head osteonecrosis. ${ }^{17}$ Other complications such as tendon rupture have also been reported. ${ }^{23}$ Lin et al reported a tendon rupture in calcific Achilles tendonitis secondary to surgery for Hoagland disease. Additionally, they also noted some other risk factors for tendon rupture such as previous surgery in the area, long-term usage of NSAIDs, and corticosteroid injection in the area ${ }^{23}$ which should be considered when using ESWT.

In the literature review, there was only one study comparing the effect rate of corticosteroid injection and ESWT. There was no statistically significant difference in the effect rate between the two methods after 1,3 , and 6 months of follow up. ${ }^{24}$ Regarding the complications and disadvantages mentioned for the methods used in the treatment of trigger finger disorder, this study investigated the effect of extracorporeal shock wave therapy in the treatment of amateur sporting participants with trigger finger. 


\section{Methods}

This study was an interventional study, with a registration number of IRCT20170915036191N2, recruiting 19 patients with trigger finger disorder referred to Physical Medicine and Rehabilitation Clinics of Isfahan University of Medical Sciences. Importantly, this trial was approved by the research ethics committee of Isfahan University of Medical Sciences, and it was conducted in accordance with the Declaration of Helsinki. After explaining the benefits, goals, and potential risks of treatment, patients were enrolled in the study and signed the informed written consent. The diagnosis was based on the accepted definition of trigger finger disorder according to European guideline ICD10. ${ }^{1}$ Importantly, amateur sporting participants in the past 2 years were recruited.

Inclusion criteria include: a) ages over 18 years old; b) trigger finger of grade one or more; c) receiving previous treatment more than 6 months ago; and d) lack of hemorrhagic diseases, pregnancies, malignancies, localized infections, heart arrhythmia, and pacemaker.

Evaluation of pain severity, severity of triggering, and functional impact of triggering was carried out using the Visual Analogue Scale, Trigger Finger Score suggested by Quinnell, ${ }^{25}$ and Quick-Disabilities of the Arm, Shoulder, and Hand (DASH) questionnaire, ${ }^{26}$ respectively, before intervention, immediately after intervention, and in 6 and 18 weeks after intervention.

Trigger Finger Score suggested by Quinnell: at the zero score, the finger is not locked, but it has crepitation during movement. A one score is given when the finger is not locked, but it has sudden movements. At the two score, the finger locking could be modified actively. A three score is given when the finger locking could be modified passively. At the four score, the finger locking is not modifiable. ${ }^{25}$

Quick-Disabilities of the Arm, Shoulder, and Hand (DASH) questionnaire: in comparison to the original 30item DASH questionnaire, the Quick-DASH is a selfreport questionnaire containing 11 items. It measures the ability of individuals to perform certain upper extremity activities to complete tasks, absorb forces, and it also measures the severity of symptoms. The Quick-DASH uses a 5-point Likert scale from which the patients select an appropriate number corresponding to their severity level or function level. ${ }^{26}$ Considering that the reliability, validity, and cross-cultural adaptation of the Quick DASH and DASH Questionnaire have been done in Persian, the Persian Quick Dash Questionnaire was used. $^{27,28}$

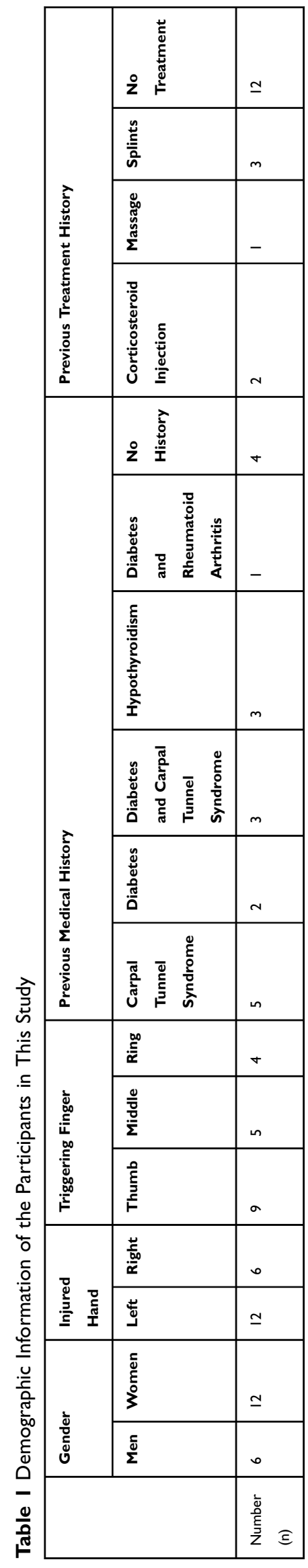


Each patient was treated with ESWT in three sessions with a 1-week interval. The treatment protocol for each session was using radial shock wave therapy with 1000 shocks, at an energy flux density of $2.1 \mathrm{bar}$ and a frequency of $15 \mathrm{~Hz}^{24}$ Moreover, the treatment protocol for using focused shock wave therapy was 500 shocks, at an energy flux density of $0.1 \mathrm{bar}$ and a frequency of $4 \mathrm{~Hz}$. Focused shock wave therapy was used directly on the nodule and the maximum tenderness site while the radial shock wave therapy was used on the peripheral tissues of the nodule. The DUOLITH SD1 Tower, Switzerland was used for intervention.

Data were analyzed using Statistical Package for the Social Sciences (SPSS) software version 17.0. Demographic data were analyzed using descriptive statistics. ANOVA was utilized to analyze changes in pain severity, severity of triggering, and functional impact of triggering during follow-ups. The level of significance was set at $0.01(P<0.01)$.

\section{Results}

Nineteen patients participated in this study, of which $27.8 \%$ had carpal tunnel syndrome, $11.1 \%$ had diabetes, and $16.7 \%$ had hypothyroidism. $27.8 \%$ of patients had trigger finger in their middle finger and $50 \%$ had this problem in their thumb. $66.7 \%$ of the participants had not received any treatment for their trigger finger. Table 1 shows the demographic information of the participants.

As Table 2 depicts, there were statistically significant differences with regard to reduction of the pain severity, severity of triggering, and functional impact of triggering before intervention, immediately after intervention, and in 6 and 18 weeks after intervention $(P<0.01)$.
The effect of ESWT on reducing severity of triggering immediately after intervention did not yield a statistically significant difference compared to before intervention $(P>0.01)$. Moreover, the effect of ESWT on reducing pain severity, severity of triggering, and functional impact of triggering in the 6 weeks after intervention did not yield a statistically significant difference compared to the 18 weeks after intervention $(P>0.01)$ (Table 3$)$.

Moreover, the F-value obtained from Pillai's Trace was 0.735 for changes in severity of triggering, 0.851 for changes in pain severity, and 0.751 for changes in functional impact of triggering during the intervention period $(P<0.01)$.

\section{Discussion}

Trigger finger disorder is a sudden release or locking of a finger during flexion or extension. Treatments for this disease are diverse and include NSAIDs, hand splints, corticosteroid injections, and percutaneous or open surgery. Regarding the complications and disadvantages mentioned for the methods used in the treatment of trigger finger disorder, the aim of this study was to investigate the effect of ESWT in the treatment of patients with trigger finger.

According to Tables 2 and 3, regarding the effect of ESWT on severity of triggering of patients with trigger finger before intervention, immediately after intervention, and in 6 and 18 weeks after intervention, it can be concluded that the mean value of severity of triggering has descended from 3.50 before the intervention to 1.75 in the 18 weeks after the intervention. However, this severity of

Table 2 The Effect of Extracorporeal Shock Wave Therapy on Reducing the Pain Severity, Severity of Triggering, and Functional Impact of Triggering Before Intervention, Immediately After Intervention, and in 6 and 18 Weeks After Intervention

\begin{tabular}{|c|c|c|c|c|}
\hline Variable & \multicolumn{2}{|l|}{ Mean \pm Standard Deviation } & F-value & $P$-value \\
\hline Severity of Triggering & $\begin{array}{l}\text { Before Intervention } \\
\text { Immediately after Intervention } \\
6 \text { Weeks after Intervention } \\
18 \text { Weeks after Intervention }\end{array}$ & $\begin{array}{l}3.50 \pm 0.96 \\
3.25 \pm 1.06 \\
2.44 \pm 1.31 \\
1.75 \pm 1.95\end{array}$ & 17.48 & $0.001 *$ \\
\hline Pain Severity & $\begin{array}{l}\text { Before Intervention } \\
\text { Immediately after Intervention } \\
6 \text { Weeks after Intervention } \\
18 \text { Weeks after Intervention }\end{array}$ & $\begin{array}{l}6.13 \pm 2.27 \\
3.31 \pm 1.77 \\
0.94 \pm 1.12 \\
0.56 \pm 1.21\end{array}$ & 50.58 & $0.001 *$ \\
\hline Functional Impact of Triggering & $\begin{array}{l}\text { Before Intervention } \\
\text { Immediately after Intervention } \\
6 \text { Weeks after Intervention } \\
\text { I8 Weeks after Intervention }\end{array}$ & $\begin{array}{l}373.44 \pm|43.3| \\
332.8| \pm| 38.96 \\
215.63 \pm \mid 53.53 \\
|67.19 \pm| 8 \mid .36\end{array}$ & 23.57 & $0.001 *$ \\
\hline
\end{tabular}

Note: $* P<0.001$. 


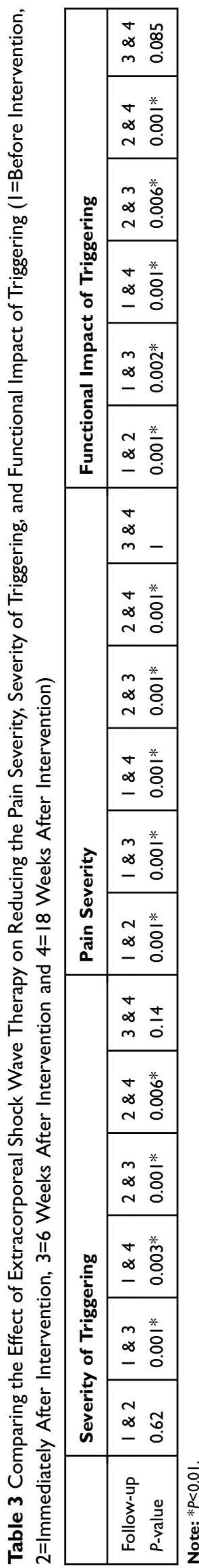

triggering was not significantly observed immediately after the intervention, and the peak effect of ESWT on reducing severity of triggering was in the 6 weeks after the intervention continuing to 18 weeks after the intervention.

Regarding the effect of ESWT on the functional impact of triggering before intervention, immediately after intervention, and in 6 and 18 weeks after intervention, it can be concluded that the mean value of the functional impact of triggering has descended from 373.44 before the intervention to 167.19 in the 18 weeks after the intervention. Table 3 demonstrates the rapid impact of ESWT on increasing the functional status of patients with trigger finger, as the 40.36 decrease in the functional impact of triggering score immediately after intervention was statistically significant and this reduction continues through the 18 weeks after intervention. Similarly, the pain severity descended from 6.13 before intervention to 0.56 in the 18 weeks after intervention. Moreover, the reduction in pain severity, by making a difference value of 2.82 in comparing before and immediately after the intervention, yielded a statistically significant difference. In a similar study by Malliaropoulos et al, the use of radial ESWT led to a pain severity reduction of 7.1 on the Visual Analogue Scale in comparing the baseline and the 1 year after intervention follow up. ${ }^{29}$ In Malliaropoulos et al's study, the mean value of pain severity was 7.8 at baseline, which is much higher than the mean value reported by the participants in our study. Considering the F-value obtained from Pillai's Trace, it can be stated that $73.5 \%$ of changes in severity of triggering, $85.1 \%$ of changes in pain severity, and $75.1 \%$ of changes in functional impact of triggering during the intervention period were due to ESWT.

In the only randomized controlled study conducted in this area, Yildirim et al compared the effects of ESWT and corticosteroid injection on reducing pain severity, severity of triggering, and functional impact of triggering. In this study, 40 patients with trigger finger were allocated into two groups of ESWT and corticosteroid injection. ${ }^{24}$ In similar findings to the results of our study, the ESWT group achieved a reduction of 4.9 in pain severity in comparing the baseline and 18 weeks after the intervention. Additionally, a significant reduction in severity of triggering and functional impact of triggering was also reported. ${ }^{24}$

Currently, ESWT is widely used in the treatment of tendinopathies. It is believed that ESWT acts by stimulating biological activity in cells leading to the creation of mechanosensitive biofeedback between the sound waves and these cells. Studies have shown that an increase in the 
angiotensin factor by ESWT results in neovascularization and appropriate vascular support of the injured tendon leading to its repair. Moreover, ESWT stimulates the synthesis of nitric oxide which will suppress the progression of inflammation. ${ }^{30-33}$ However, the effectiveness of ESWT depends on several factors including the location of applying pressure, the energy flux density, the overall energy, adherence to the principles of shockwave production, and the device itself. ${ }^{34}$ Moreover, the application of concomitant local anesthesia is demonstrated to reduce the effectiveness of ESWT; ${ }^{35,36}$ therefore, the ultrasound gel was the only conductive gel used in this study. It should be also noted that the protocol used by Yildirim et al was used in this study. Two of the limitations of this study were the lack of randomization in selecting the sample population and the lack of a control group. Moreover, the number of selected samples was relatively small. It is suggested that these constraints be considered in future studies.

It seems that ESWT leads to a reduction in pain severity, severity of triggering, and functional impact of triggering. This reduction persisted until the 18 th week after the intervention. It is recommended to use extracorporeal shock wave therapy in terms of a non-invasive intervention with no significant complications for patients with trigger finger.

\section{Data Sharing Statement}

The authors are willing to share individual de-identified participant data of demographic information, pain severity, severity of triggering, and functional impact of triggering before intervention, immediately after intervention, and in 6 and 18weeks after intervention on demand. The clinical trial data will be made available for 5 years after the publication date of this study by contacting the corresponding author via email. No other study-related documents will be made available by the authors.

\section{Disclosure}

The authors report no conflicts of interest in this work.

\section{References}

1. Huisstede BM, Hoogvliet P, Coert JH, Fridén J. Multidisciplinary consensus guideline for managing trigger finger: results from the european HANDGUIDE study. Phys Ther. 2014;94(10):1421-1433. doi:10.2522/ptj.20130135

2. Frontera WR, Silver JK, Rizzo TD. Essentials of Physical Medicine and Rehabilitation: Musculoskeletal Disorders, Pain, and Rehabilitation. Saunders/Elsevier; 2008.

3. Bonnici A, Spencer J. A survey of 'trigger finger'in adults. J Hand Surg Eur Vol. 1988;13(2):202-203. doi:10.1016/0266-7681(88)90139-8
4. Moore JS. Flexor tendon entrapment of the digits (trigger finger and trigger thumb). J Occup Environ Med. 2000;42(5):526-545. doi:10.1097/00043764-200005000-00012

5. Ryzewicz M, Wolf JM. Trigger digits: principles, management, and complications. J Hand Surg Am. 2006;31(1):135-146. doi:10.1016/j. jhsa.2005.10.013

6. Cagliero E, Apruzzese W, Perlmutter GS, Nathan DM. Musculoskeletal disorders of the hand and shoulder in patients with diabetes mellitus. $\mathrm{Am}$ J Med. 2002;112(6):487-490. doi:10.1016/S0002-9343(02)01045-8

7. Koh S, Nakamura S, Hattori T, Hirata H. Trigger digits in diabetes: their incidence and characteristics. J Hand Surg Eur Vol. 2010;35 (4):302-305. doi:10.1177/1753193409341103

8. Rhoades CE, Gelberman RH, Manjarris JF. Stenosing tenosynovitis of the fingers and thumb: results of a prospective trial of steroid injection and splinting. Clin Orthop Relat Res. 1984;190:236-238.

9. Dehghani M, Alerasoul S, Saadatpoor L, Alerasoul M. Comparing the positive results of carpal tunnel syndrome surgery in two groups of patients with and without splint. J Isfahan Med Sch. 2014;31 (267):2155-2161.

10. Dehghani M, Zarezadeh A, Shemshaki H, Moezi M, Nourbakhsh M. Hour glass constriction in advanced carpal tunnel syndrome. Int J Prev Med. 2013;4(4):438.

11. Vahdatpour B, Raissi G, Hollisaz M. Study of the ulnar nerve compromise at the wrist of patients with carpal tunnel syndrome. Electromyogr Clin Neurophysiol. 2007;47(3):183-186.

12. Dehghani M, Shemshaki H, Eshaghi MA, Teimouri M. Diagnostic accuracy of preoperative clinical examination in upper limb injuries. J Emerg Trauma Shock. 2011;4(4):461.

13. Wu W-T, Chang K-V, Mezian K, Naňka O, Lin C-P ÖL. Basis of shoulder nerve entrapment syndrome: an ultrasonographic study exploring factors influencing cross-sectional area of the suprascapular nerve. Front Neurol. 2018;9:902. doi:10.3389/fneur.2018.00902

14. Wang J-C, Chang K-V, Wu W-T, Han D-S ÖL. Ultrasound-guided standard vs dual-target subacromial corticosteroid injections for shoulder impingement syndrome: a randomized controlled trial. Arch Phys Med Rehabil. 2019;100(11):2119-2128. doi:10.1016/j. apmr.2019.04.016

15. Patel M, Bassini L. Trigger fingers and thumb: when to splint, inject, or operate. J Hand Surg Am. 1992;17(1):110-113. doi:10.1016/03635023(92)90124-8

16. Newport ML, Lane LB, Stuchin SA. Treatment of trigger finger by steroid injection. J Hand Surg Am. 1990;15(5):748-750. doi:10.1016/ 0363-5023(90)90149-L

17. Braddom RL. Physical Medicine and Rehabilitation. Elsevier Health Sciences; 2010.

18. Cacchio A, Giordano L, Colafarina O, et al. Extracorporeal shock-wave therapy compared with surgery for hypertrophic long-bone nonunions. J Bone Joint Surg. 2009;91(11):2589-2597. doi:10.2106/JBJS.H.00841

19. Mouzopoulos G, Stamatakos M, Mouzopoulos D, Tzurbakis M. Extracorporeal shock wave treatment for shoulder calcific tendonitis: a systematic review. Skeletal Radiol. 2007;36(9):803-811. doi:10.10 07/s00256-007-0297-3

20. Stasinopoulos D, Johnson M. Effectiveness of extracorporeal shock wave therapy for tennis elbow (lateral epicondylitis). $\mathrm{Br} J$ Sports Med. 2005;39(3):132-136. doi:10.1136/bjsm.2004.015545

21. Vahdatpour B, Sajadieh S, Bateni V, Karami M, Sajjadieh H. Extracorporeal shock wave therapy in patients with plantar fasciitis. A randomized, placebo-controlled trial with ultrasonographic and subjective outcome assessments. Res Med Sci. 2012;17(9):834.

22. Vahdatpour B, Taheri P, Zade AZ, Moradian S. Efficacy of extracorporeal shockwave therapy in frozen shoulder. Int J Prev Med. 2014;5(7):875.

23. Lin T-C, Lin C-Y, Chou C-L, Chiu C-M. Achilles tendon tear following shock wave therapy for calcific tendinopathy of the Achilles tendon: a case report. Phys Ther Sport. 2012;13(3):189-192. doi:10. 1016/j.ptsp.2011.08.002 
24. Yildirim P, Gultekin A, Yildirim A, Karahan A, Tok F. Extracorporeal shock wave therapy versus corticosteroid injection in the treatment of trigger finger: a randomized controlled study. J Hand Surg Eur Vol. 2016;41(9):977-983. doi:10.1177/1753193415622733.

25. Quinnell R. Conservative management of trigger finger. Practitioner. 1980;224(1340):187-190.

26. Luc D. The DASH questionnaire and score in the evaluation of hand and wrist disorders. Acta Orthop Belg. 2008;74:575-581.

27. Ebrahimzadeh MH, Moradi A, Vahedi E, Kachooei AR, Birjandinejad A. Validity and reliability of the Persian version of shortened disabilities of the arm, shoulder and hand questionnaire (quick-DASH). Int J Prev Med. 2015;6:59.

28. Mousavi SJ, Parnianpour M, Abedi M, et al. Cultural adaptation and validation of the persian version of the disabilities of the arm, shoulder and hand (DASH) outcome measure. Clin Rehabil. 2008;22(8):749-757. doi:10.1177/0269215508085821

29. Malliaropoulos N, Jury R, Pyne D, et al. Radial extracorporeal shockwave therapy for the treatment of finger tenosynovitis (trigger digit). Open Access J Sports Med. 2016;7:143. doi:10.2147/OAJSM.S108126

30. Ciampa AR, de Prati AC, Amelio E, et al. Nitric oxide mediates antiinflammatory action of extracorporeal shock waves. FEBS Lett. 2005;579(30):6839-6845. doi:10.1016/j.febslet.2005.11.023
31. Orhan Z, Ozturan K, Guven A, Cam K. The effect of extracorporeal shock waves on a rat model of injury to tendo Achillis. Bone Jt J. 2004;86(4):613-618.

32. Wang CJ, Wang FS, Yang KD, et al. Shock wave therapy induces neovascularization at the tendon-bone junction. A study in rabbits. J Orthop Res. 2003;21(6):984-989. doi:10.1016/S0736-0266(03)00104-9

33. Hsu RWW, Hsu WH, Tai CL, Lee KF. Effect of shock-wave therapy on patellar tendinopathy in a rabbit model. J Orthop Res. 2004;22 (1):221-227. doi:10.1016/S0736-0266(03)00138-4

34. Baloglu I, Ozsoy M, Aydınok H, Lok V. Extracorporeal shock wave therapy in orthopedics and traumatology. Off J Turk Soc Orthop Traumatol. 2005;4:33-49.

35. Pettrone FA, McCall BR. Extracorporeal shock wave therapy without local anesthesia for chronic lateral epicondylitis. JBJS. 2005;87 (6):1297-1304.

36. Rompe JD, Meurer A, Nafe B, Hofmann A, Gerdesmeyer L. Repetitive low-energy shock wave application without local anesthesia is more efficient than repetitive low-energy shock wave application with local anesthesia in the treatment of chronic plantar fasciitis. J Orthop Res. 2005;23(4):931-941. doi:10.1016/j.orthres.2005.09.003

\section{Publish your work in this journal}

Open Access Journal of Sports Medicine is an international, peerreviewed, open access journal publishing original research, reports, reviews and commentaries on all areas of sports medicine. The manuscript management system is completely online and includes a very quick and fair peer-review system. Visit http://www.dovepress. com/testimonials.php to read real quotes from published authors. 